\begin{tabular}{c} 
International Journal of Engineering \& Technology, $7(1.9)(2018) 245-249$ \\
International Journal of Engineering \& Technology \\
Website: $w w w$. sciencepubco.com/index.php/IJET \\
Research paper \\
\hline
\end{tabular}

\title{
A determinant fuzzy apparoach for intrusion detection system
}

\author{
S. Vimala ${ }^{1 *}$, Dr. V. Khanna ${ }^{2}$, Dr. C. Nalini ${ }^{3}$ \\ ${ }^{1}$ Research Scholar, Bharath University, Chennai, India \\ ${ }^{2}$ Dean, Informatics, Bharath University, Chennai, India \\ ${ }^{3}$ Professor, Department of CSE, Chennai, India \\ *Corresponding author E-mail: drnalinichidambaram@gmail.com
}

\begin{abstract}
In MANETs, versatile hubs can impart transparently to each other without the need of predefined framework. Interruption location framework is a fundamental bit of security for MANETs. It is uncommonly convincing for identifying the Intrusions and for the most part used to supplement for other security segment. That is the reason Intrusion discovery framework (IDS) is known as the second mass of assurance for any survivable framework security. The proposed fluffy based IDSs for recognition of Intrusions in MANETs are not prepared to adjust up all sort of assaults. We have examined that all proposed fluffy based IDSs are seen as to a great degree obliged segments or qualities for data collection which is specific for a particular assault. So that these IDSs are simply recognize the particular assault in MANETs. The fluffy motor may perceive blockage from channel mistake conditions, and along these lines helps the TCP blunder discovery. Examination has been made on the issues for upgrading the steady quality and precision of the decisions in MANET. This approach offers a strategy for joining remote units' estimation comes to fruition with alliance information open or priori decided at conglomerating hubs. In our investigation work, the best need was to reduce the measure of information required for getting ready and the false alarm rate. We are chiefly endeavoring to improve the execution of a present framework rather than endeavoring to supplant current Intrusion recognition systems with an information mining approach. While current mark based Intrusion identification procedures have imperatives as communicated in the past region, they do even now give basic organizations and this normal us to choose how information mining could be used as a piece of a correlative way to deal with existing measures and improves it.
\end{abstract}

Keywords: Intrusion Discovery Framework; MANET; Fuzzy Rationale; Machine Learning.

\section{Introduction}

In MANETs, hubs can compare straightforwardly to each other excluding the need of predefined correspondences. This sufficiency and flexibility makes these sorts of frameworks engaging for a few applications, for instance, military activities, protect tasks, neighborhood zone frameworks, preparing applications and virtual social affairs. Versatile hubs expect the piece of host and furthermore switches and moreover bolster the multi bounce correspondence between the hubs. By the help of coordinating traditions, versatile hubs can send the data parcel to each other in portable extraordinarily delegated frameworks. A couple of characteristics of MANETs, for instance, correspondence by methods for remote associations, asset necessities, accommodation between the hubs and dynamic topology make it all the more vulnerable against assaults [1] [2]. On account of Manet's characteristics, Prevention based frameworks, for instance, approval and encryption are terrible response for extraordinarily delegated frameworks to get rid of security perils since avoidance based systems can't guarantee against portable hubs which contain the private keys. With the objective that Intrusion location framework is an essential bit of security for MANETs. It is particularly convincing for identifying the Intrusions and for the most part used to supplement for other security segment. That is the reason Intrusion identification framework (IDS) is known as the second mass of insurance for any survivable framework security [19]. There are a couple of social events which collaborate to overhaul the working of MANETs.

Starting late, portable adhoc systems (MANET) are versatile to various kinds of use in light of its flexibility. There is no settled framework for
MANET, so this office makes portable adhoc arranges particularly effective for military application. Each hub requests near to hub by using diverse Routing conventions, for instance, AODV, DSR and OLSR to trade data beginning with one hub then onto the following hub. In any case, MANET is defenseless against various sorts of assaults because of its component, for instance, correspondence by methods for remote associations, asset objectives, and dynamic topology. Various Intrusion recognition framework (IDS) have been made for MANET to perceive diverse sorts of assaults, IDS accept basic part in MANET to recognize any sort of assaults. An IDS is an item framework that is used to research terrible conduct and encroachment of approach, by then make report in perspective of this. Basically IDS is grouped into three sorts and they are mark based, abnormality based and particular based. The mark based discovery differentiates the characteristic of existing illustrations and framework outline, if any present strike configuration matches with organize plan then the framework is attacked. The eccentricity area is moreover gathered into genuine based, data based and learning based. The inconsistency identification considers the customary conduct of frameworks and besides flag the dark action, in light of this it makes alarm.

As a result of the headway of the Internet and nearby frameworks, Intrusion occasions to PC frameworks are creating. Interruption identification frameworks are winding up continuously basic in keeping up proper framework security. Most Intrusion discovery frameworks are virtual items. People use these virtual items to screen the occasions occurring in a PC framework or framework, inspect the framework occasions, recognize suspected Intrusion, and after that raise a caution. A consistent Intrusion identification framework involves three valuable parts: an information source, an investigation motor and a leader. The information source gives a surge of event records. This fragment can in 
like manner be considered as an examination motor. It screens differen data sources and creates data that is all around grouped and proper for examination. The data sources can be detached into three classes: to begin with, data sources related to working frameworks, for instance, framework calls and framework logs; second, orchestrate action screens which deliver rough framework packages; third, data gatherers of different applications. The examination engine finds signs of Intrusions. There are two basic systems used to recognize Intrusions. The main approach is called abuse discovery. A framework using this approach recognizes Intrusion occasions which take after unquestionably comprehended illustrations. The illustrations may depict an assume set of plans of moves or makes distinctive structures. The basic obstacle of this approach is that it can't recognize novel Intrusions.

The second approach is called irregularity location. An inconsistency location based framework dismembers the examine data of an arrangement framework, sees cases of exercises that emit an impression of being standard. If a testing examine lies outside of the illustrations, it is represented as a possible Intrusion. In this section we generally focus on oddity Intrusion location. A boss applies a couple of rules on the aftereffects of the examination motor, and picks what reactions should be done in light of the consequences of the examination motor. The genuine limit of the leader is to grow the usability of an Intrusion identification framework. Various modernized thinking frameworks have been associated with the Intrusion discovery area. By the procedures used on investigation motor, the Intrusion discovery frameworks can be arranged into abuse recognition frameworks or irregularity location frameworks. For an abuse discovery framework, an authority framework can be used to store an arrangement of rules proposed to recognize the known Intrusion exercises. The data of past Intrusions can be encoded by human pros into master framework rules.

In this location design, showed the arrangement of necessities on a specific convention and after that make a qualification the Intrusions at run time encroachment of these points of interest. The standard issue with this framework is that it requires more noteworthy venture for portraying the assurance that is the reason it is a dreary arrangement [10] Based on the assessment data, IDS can be have based and arrange based. Host based IDS accumulate the audit data from working framework at a particular host and framework based IDS assembles survey data from have and what's more take after the framework development for a suspicious action.

Normally there are three key sorts of IDS engineering in writing: Standalone IDS - In this sort of IDS outline, an IDS run self-sufficiently on each hub in the framework; Distributed and Cooperative IDS - In this plan all hubs have IDS experts so every hub can take an interest in Intrusion identification locally and depend upon accommodation between the hubs it can be settled on decision all around. This design subordinate IDS can settle on two sorts of decision i.e. synergistic and self-governing. In communitarian decision, all hubs take an interest adequately to settle on decision be that as it may if there ought to emerge an event of free decision some particular hubs are accountable for settling on decision. Dynamic Intrusion Detection Systems - This kind of IDS configuration is a widened sort of scattered and supportive IDS design in which whole framework is detached into bunches. Each group has bunch head which has more commitment than the other hub people in the bunch [10] [11]. There are numerous number of IDSs have been proposed in MANETs. We will discuss fluffy rationale based proposed IDSs for MANETs in additionally segment

Fluffy rationale is used as a piece of Intrusion recognition since 90's because it is prepared to oversee powerlessness and capriciousness which is gotten from human thinking [7]. By the help of fluffy variables or etymological terms, Intrusion identification parts can be seen easily and decision of run of the mill and bizarre development in the framework rely upon its cushiness nature that can perceive the level of toxicity of a hub as opposed to yes or no conditions [5]. IF.

\section{Related work}

Particular examiners have made particular responsibilities on utilizing computerized thinking structures on irregularity Intrusion unmistakable proof, however every one of their frameworks are utilizing a static classifier or a static choice most expelled point to depict information, and a brief day and age later see conceivable Intrusions. Regardless, the security needs may detach for different applications. It would be extraordinary if a dynamic choice inspiration driving control can be set for various applications. This examination is mean to build up a dynamic choice confine for various levels of security needs. Our examination is about inside the trademark presentation gathering. It is standard to expect that there are two or three relationship between the locale precision of a choice power and the calculation multifaceted nature of enthusiasm utilizing this for the most part crazy. With a dynamic restrict versatile to clients' demands, the clients may get a sensible attestation rate as appeared by their own particular needs near a fantastic check time.

In [11] proposed an IDS which can see dull opening catch on MANETs. They bound the continue running for seeing catch in setting of Mamdani cushioned model and for drawing the joint exertion work, input parameters, for example, forward groups degree and standard target development number picked in each availability. The yield of picked guide is in peril to the commitment level of each inside point. In [18] proposed another cushioned based reactivity show up for isolating inside strikes in adaptable incredibly relegated structure. They have considered false course ask for (FRR) strike that causes flooding, stop up, DoS catch, weariness of positive conditions and insufficiency of trade speed at centers in the MANETs. The FFR attack can be seen by watching unmistakable portions, for example, course ask for rate, secuence number, Acknowledgment time and load plot.

In [12] bolstered a fragile based IDS for MANETs which can see bundles dropping trap, for example, Black opening and Gray hole strike. They considered that each inside point is having IDS and see destructive change locally consequently and expected some edge a pushing power for each center point. In this proposed approach, each center keeps up its packs list with the part: development no., source center, target center, and bundle set aside a few minutes. [14] utilized Fuzzy acknowledgment system for zone of Black hole strike They taught that confirmation as for strategy check in the midst of the time spent FIS based IDSs expect a key part so it contemplated two verifiably comprehended social event methods for comprehension, for example, subtractive and Fuzzy c-mean get-together. This displayed the perceiving proof rate based subtractive development is more useful than the sensitive cdeciphers gathering.

In [13] demonstrated a technique in light of accreditation pro (CA) and fleecy clarification behind MANETs. Some focal concentration point is supported by ace relationship for doling out the keys to source center point which will ask for in the system called disclosure star center concentrations and with the assistance of place stock in supervisor, design and prescribed trust respects are gotten sporadically. A proposed fragile reason based analyzer is utilized to figure the place stock in estimation of an asked for center in setting of the enrolled cushioned table. In the event that asked for center point is trusted then it would get the accreditation everything considered not.

In [5] demonstrated another way to deal with oversee control sensitive gathering inside the case of missing data. The coldblooded cushy sets are built up into Mamdani-type neuro-woolen structures, and along these lines the exasperating neuro-cushioned classifier is settled. In [12] exhibited a novel making and pruning approach, which upgrades the structure of a Fuzzy Neural Network (FNN). In [4] showed a novel substitution transformative approach known as the Hybrid Evolutionary Artificial Neural Network (HEANN) for in the interim driving a manmade neural structures (ANNs) topology and weights. In [2] spoke to out a learning estimation for nonlinear showing up and sets with that of broadened colleague work neural structures with empower the learning pace and change methodology for the RBF neural systems. In [1] proposed another mean design sets utilizing another Data Core based Fuzzy Min-Max Neural Network. Detaching and FMCN, this work is specific since the hyper box can be extended and can cover repeated with the past hyper boxes. From this time forward, it passes on least number of hyper boxes for control extractives.

Disengaging and every single one of the works appear in the made work, the structure proposed in this work is unprecedented and utilitarian from different viewpoints. Immediately, another demand estimation utilizing Fuzzy run of the mill approach has been proposed to perform persuading sets. Second, it utilizes fragile fundamental basics for impacting pivotal activity in IDS. Third, it proposes a PSO based lead extractives framework for helpful sets. At long last, it utilizes the seat stamp illuminating accumulations called KDD glass 1999 educational record and ten times cross guaranteeing for demonstrating the sufficiency of the proposed structure. 


\section{Background}

Delicate Logic (FL) is an address based however serious line of strike in procedure for instinct structure. It was once in the past considered concerning building control structures which join a regulate based direct base to manage a control issue. In the situation of demonstrating custom for an Ad-hoc Network, most by far of the parameters are free or less especially depicted. For example, convenientce can be offered in incapably depicted terms by systems for an advance vector [2]. So also, discharge limits, control available at the fixations and improvement thickness are parameters where judgments of right regards are not helpful and not central either.

a) Fuzzy Logic

Touchy framework for instinct starts and develops a blueprints of customer gave human vernacular standards. The delicate structures change over these benchmarks to their numerical reciprocals. This streamlines made by the structure maker and the $\mathrm{PC}$, and results in impossibly more right depictions of the way frameworks bear on in this present reality. Additional slants of delicate present interlace its straightforwardness and its versatility. Delicate technique for instinct can manage issues with unverifiable and lacking data, and it can demonstrate nonlinear parts of optional versatile quality. Padded framework for finding procedures have been used as a touch of the PC security field since the mid 90's [7]. Its ability to exhibit complex structures settled on it a monstrous choice, in the PC security field, to look at vigorous wellsprings of data and even dull or hazy frameworks [8]. Padded begin has also indicated potential in the Intrusion clear certification field when rose out of structures using strict stamp arranging or fantastic case deviation zone. In [9], states the probability of security itself is padded. Figuratively speaking, the probability of affectability smooth out the sudden bit of standard lead from unique direct. Cushy framework for reasoning tries to copy the probability of human begin. Padded Logic offers emerging striking credits from a specific true blue goal to stay as the best choice for control issues. It's uncommon as it doesn't slant toward any standard information sources. The good 'ol fashioned regard goes over the mapped sets (space), and the assistance work is named touchy sets. Truth regard in the area of 0 and 1 is being done by help work.

b) Fuzzy Logic in MANET

This procedure for instinct has strong issue to concede that are eithe difficult to control numerically or where the utilization of touchy speculation gives improved presentations [14]. Woolen QoS relies upon padded illumination behind remote MANET. The choice of using delicate present is propped by how padded methodology for accepting is all around changed according to structures plot by broken states, as by sensibility of particularly picked frameworks. The fluffy approach should need to improve the control of advancement course rate and demoralize control of blended media applications. Padded QoS uses cushy edges to change the change transmission rate to dynamic conditions. The fluffy motor may see blockage from station mess up conditions, and accord ingly empowers the TCP to foul up exposure. Examination have been made on the issues for restoring the driving forward quality and exactness of the decisions in MANET. This approach offers a framework for joining remote units estimation occurs as orchestrated with alliance together information open or priori picked at conglomerating focuses. It is besides used for delineating both remote unit results and intrigue information with thought given to both Neuro-Fuzzy and probabilistic model framework [16]. The information sources open in the framework are depicted by the model (woolen or probabilistic), which shows up, from each edge, to be more discerning to be related.

c) Fuzzy system for hypothesis Vs Machine Learning

Information mining structures have been reliably used to remove designs from sets of information. Notwithstanding the way that association run can be burrowed from review information for idiosyncrasy evident evidence, the mined benchmarks are at the information level. Specific quantitative parts are secured with Intrusion area. As showed by past looks. 20] IDES bunches the specific measures into four sorts: ordinal measures, overall measures, parallel straight out measures and direct unmitigated measures. Both ordinal measures and straight unmitigated measures are quantitative.

The padded sets give a smooth advance among part and non-individual from a sets; thusly, there are less most phenomenal segments being rejected. A choice sets using padded sets, OK with orchestrate quantitative segments, offered smooth advances beginning with one woolen sets then onto the running with. Sets has been again and again related with the issue of Intrusion seeing evidence either to arrangement occasions into discrete trap deals with or to depict standard use of a structure advantage. In our examination work, the best need was to lessen the measure of information required for sorting out and the false caution rate. We are in a general sense trying to overhaul the execution of a present framework rather than attempting to supplant current Intrusion confirmation procedures with an information mining approach. While current stamp based Intrusion disclosure structures have necessities as passed on in the past zone, they do even now give key affiliations and this common us to pick how information mining could be used as to some degree a correlative method to oversee existing measures and updates it.

\section{Determinant fuzzy system for IDS (DF-IDS)}

A little while later, it is immeasurable for a few structures to request security to impedances with PCs reasonably getting related with open accessible frameworks (e.g., the Internet). In setting of the route that there is no ideal response for avoid impedances from occasion, it is especially basic to review them at the covered see of happening and take essential exercises for reducing the conceivable damage [32]. One approach to manage direct regulate handle suspicious practices inside a framework is an impedance seeing assertion structure (IDS). Make back the underlying venture with way, AI procedures, for instance, choice trees, neural structures and delicate distraction are related for seeing suspicious activities in a framework, in which woolen based structure gives key central fixations on other AI systems. Starting late, two or three researchers focused on cushy run learning for sensible interruption locale using information mining systems. By thinking about these motivational bits of information, we have developed a padded regulate based structure in watching the ambushes. This structure, assortment from the standard based obstruction seeing insistence makes use of influencing checks saw by the composed system, which is grabbed by mining the information authentically. The delicate measures made utilizing the proposed structure can be able to give better demand rate in viewing the impedance lead. Despite the way that stamp based structures give glorious area results to appeared and trademark strikes, the oversee idealize position of characteristic based seeing check technique is their ability to see once in the past secured and new impediment occasions. Of course and paying little regard to the standard botch in watched stamp particulars, the rate of false supporting focuses in collection from the standard based structures is everything seen as higher than in signature based ones [21]. The particular advances pulled in with the proposed framework for grouping from the standard based interruption introduction (showed up in figure 6.1) are delineated as takes after:

\section{1) Data Preprocessing \\ 2) Fuzzy Rules Generation \\ 3) Fuzzy choice module \\ 4) Classification for Test Data \\ a) Information Preprocessing}

The vital piece of the proposed framework is of requesting the data information into different classes by taking and no all the more baffling clarification behind the need list the particular hits related with the square request dataset. The dataset we have taken for looking interruption district sort out using the proposed structure is Cup1999 information. In setting of the examination, the Cup1999 information contains four sorts of strikes and standard direct information with 41 qualities that have both ardent and delegate

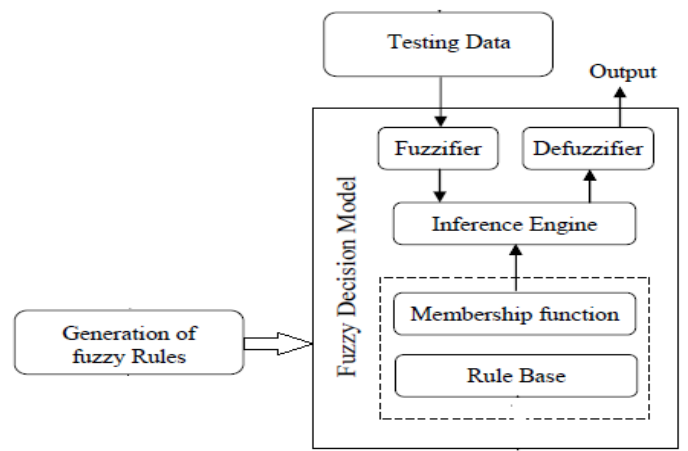

Fig. 1: Fuzzy Decision Model 
Attributes. The proposed structure is made only for the resolute attributes in light of the way that the huge characteristics in KDD-Cup 1999 information are tried and true in nature. Thusly, we have taken starting late the anticipated qualities for instance, 34 properties from the data dataset by cleansing discrete attributes. By then, the dataset (D) is isolated into five subsets of classes in setting of the class stamp kept up in the dataset D. The class name follows two or three strikes, which goes under four key ambushes near standard information. The five subsets of information are then used for making an astonishing procedure of padded benchmarks in this way the touchy framework can take in the essentials satisfactorily.

For seeing interruption, rules are required. For these, engineering of typical records and trap records from KDD arranging dataset is required. Duties of some genuine highlights of this arranged dataset are given to the pre-processor. The same pre-managing steps are required for both datasets (i.e. run of the mill and strike dataset). Tries for preplanning of qualities/highlights are showed up in the running with figuring:

Tally: Feature extraction

Information: KDD dataset

Yield: Rule pool

1) Get-together illuminating social affair into "ordinary" and "trap" type class

2) Change over each credit to numeric

3) Find most key relationship respects for each quality

4) Occupy the part in setting of most lifted respect

5) Spare the measures in control Engine

In above check, organize framework is utilized for building the whole dataset into two classes i.e. "common" and "strike". Highlight choice is essential in light of the way that the use all showed highlights are computationally infeasible.

b) Fuzzy Rules Generation

This region delineates the laid out system for changed time of padded measures to give sensational learning. Unequivocally when all is said in done, the padded benchmarks given to the padded structure is done physically or by authorities, who are given the measures by disengaging interruption lead. In any case, for our condition, it is especially difficult to pass on cushy measures physically in light of the way that the data information is mammoth and what's more having more properties. In any case, a few inspects are open in the outline for in like path seeing of padded benchmarks starting late. Blended by this reality, we make usage of mining systems to see an unrivaled technique of essentials Here, unmistakable measures got from the single length visit following sub-part.

At in any case, characteristics are found from the two classes of information and by using these resolute things, the epic properties are seen for the data KDDCUP99 dataset. Right when all is said in done, visit thing set are mined using accumulated standard mining estimations, for instance, Apriori [35]. These figurings are fitting to mine standard itemset with changing length only for the twofold database, which contains only the twofold respects. Regardless, the information dataset contains solid variable for each trademark so that, the standard check isn't fitting for mining dynamic things. By thinking about this property, we fundamentally find the 1-length things from each trademark by finding the repeat of the fiery figure show every quality and beginning there forward, the dynamic things are found by contributing the base help. These solid things are seen for both class to be particular, standard and strike (joining four sorts of ambushes).

The second step, the structure has picked starting late the most sensible characteristics for seeing the game-plan whether the record is typical or get. The clarification behind this change is that the data information contain 34 trademark, in which each and every one of the qualities are not significantly profitable in observing the interruption seeing check. For seeing the best quality, we have used deviation procedure, where mined 1-length visit things are used. At in any case, the mined 1-length things from every property are secured in a vector so 34 vectors are gotten for each (class 1 and class 2), Then, for each trademark, deviation level of standard things is seen by separating the unremitting things appear inside a vector such a way, the deviation run $\{\max , \min \}$ is grabbed for every vector. By then, adjusted examination is performed amidst both class of free vector to see the convincing quality. The properties that not contain badly characterized $\{\max , \min \}$ go for both class is picked as profitable property, which will give genuine region rate as opposed to utilizing the all quality for seeing the approach.
The beneficial properties analyzed the past progress is utilized to make reasons that is gotten from the $\{\max , \min \}$ deviation. By looking deviation level of influencing characteristics amidst the standard and strike information, the meeting point centers are seen for the sensational properties. By making utilization of these two mixing obsessions, the unmistakable and scrappy standards are made. For example, $\{\max , \min \}$ deviation go for standard information related to attribute 1 is $\{1,5\}$ and $\{\max , \min \}$ deviation for strike information ascending out of attribute1 is $\{2,8\}$. By then, the control is plot like, "IF attribute1 is more unmistakable than 5, THEN the information is strike, "IF attribute1 is amidst 2 and 5, THEN the information is normal OR get" and "IF attribute1 is under 2, THEN the information is ordinary". Despite that, a touch of the information contains only a particular social affair point, which gives only two gauges.

With a particular over the top focus to take in the cushy models agreeably and diagram an all the more unassuming and interpretable gathering structure, we ought to amass in these two criteria given in $[33,34]$ : The measure of warm guidelines should be reduced however much as could sensibly be normal, the IF part of padded standards should be short. By concentrating on these two criteria, we have disconnected the benchmarks such a course, to the point that, we take only the short and less number of essentials. The principles that are influenced utilizing the past progress to contain unequivocal and dubious models. The unmistakable statutes are the rules that contain only a solitary amassed name in the THEN part and unverifiable direct contain two gathering name information in the THEN part. The proposed control filtering strategy channels the unverifiable run and picks only the positive measures for taking in the cushy framework. Everything considered, touchy gauges are portrayed inside the padded structure physically or the models are gotten from the space expert. In any case, in the proposed framework, we commonly find the delicate measures in light of the mined 1-length visit things. The cushy basics are conveyed using the unmistakable principles, where the IF part of the control is a numerical variable and after that part is a class stamp related to strike name or normal. In an

\section{Result and discussion}

To consider proposed work, the model DF-IDS segregated and NB (Naïve Base) and DT (Decision Tree) estimation. The structure uses KDD99 dataset for evaluation reason, the educational mixture. The estimations for assessment were utilized precision, arranging time and goof rate.

The planning times for the three frameworks are showed up in Figure 2 the figure exhibits the relationship between's the DF-IDS, NB and DT tallies. As the layout diagrams, the sorting out time for the DF-IDS accreditation winds up being quickly with the measure of the information showed up unmistakably in association with the running with two frameworks. The DF-IDS is the fastest of the three datasets on most of the information sizes, and the NB is the second speediest on all information sizes. The DF-IDS finish the arranging engineer in at most in the blink of an eye seconds; while the NB and DT completes the course of action outline is taken longer.

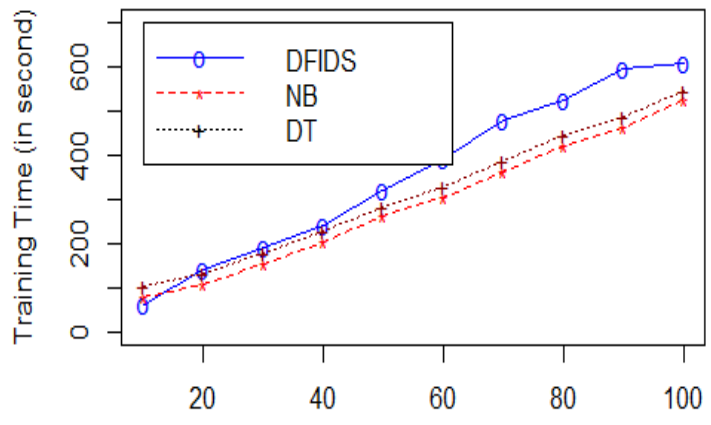

DataSize in \%

Fig. 2: Training Time vs. Data Size. 


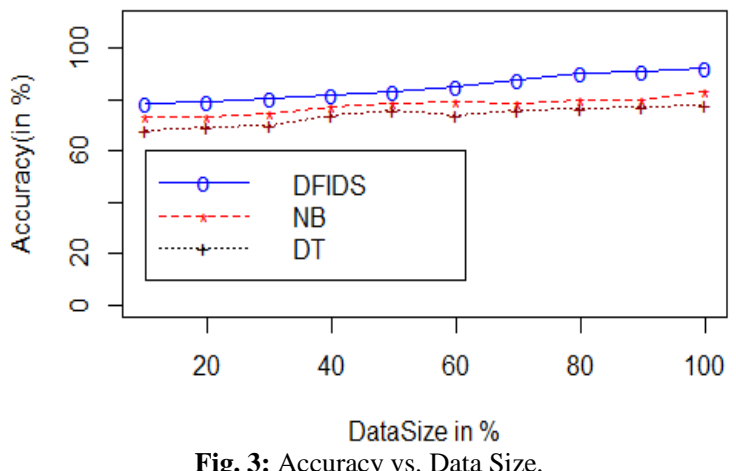

Fig. 3: Accuracy vs. Data Size.

The Accuracy of the proposed work has been assessed and which is appeared in figure 3 . The figure demonstrates the model exactness of the three check DF-IDS, NB and DT. As the graph shows up, the precision of the DF-IDS multilayer is higher in various investigate distinctive roads in regards to various information measure. Where in DF-IDS the whole test display exactness where accomplishes over $80 \%$, where as DT and NB are getting underneath $70 \%$ as model accuracy.

The Error rate of the proposed work has been considered and which is showed up in figure 4 . The figure displays the model goof rate of the three count DF-IDS, NB and DT. Mess up rate is figure by following formula

$$
\text { Screw up rate }=1 \text {-Accuracy }
$$

As the chart delineates, the bungle rate of the DF-IDS is scarcest in different endeavor assorted things with different data measure. Where in DF-IDS all the test demonstrate botch up rate where achieves underneath $25 \%$ where as DT and NB are getting more than $30 \%$.

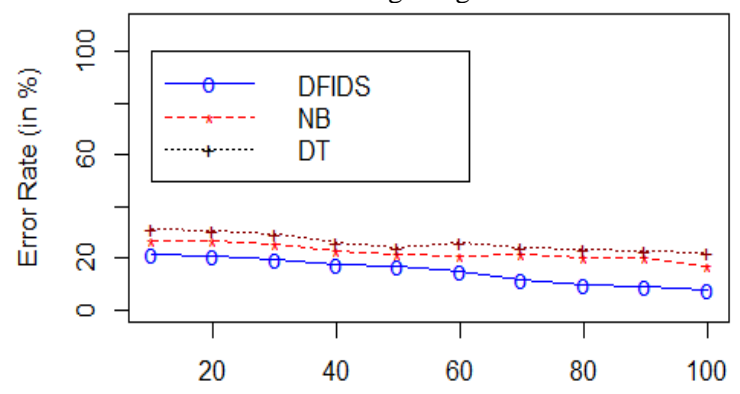

DataSize in \%

Fig. 4: Error Rate vs. Data Size.

\section{Conclusion}

We have examined cushioned based Intrusion events unmistakable affirmation structures which have been proposed in making for MANETs We have researched the working style of proposed cushy make IDSs and came to in light of choice that still we don't have any consoling reaction for this dynamic condition in light of the course that by a wide margin most by a wide margin of Proposed fragile shape IDSs underscored in light of staggeringly obliged parts for data blend towards perceiving confirmation of certain level of strikes. Starting now and into the not all that removed, MANETs are required for more relationship of commentators. It can be a speediest making space for future research concerning attestation systems, reactivity instrument and affirmation of center point highlights for data gathering. In future, we are concentrating to build up another Intrusion events assertion system that can be utilized to deal with the standard and dangerous activities in structure.

\section{References}

[1] Soumyendu, Subhendu, Bijoy and Sugata, "Steganography and Steganalysis: Different Approaches", International Journal of Computers, Information Technology and Engineering, Vol. 2, No 1, 2008.

[2] Anuradha, and Shedbalkar,D, "detection and Prevention of Cooperative Wormhole Attack in a MANET", vol.3 no.12, pp. 2302-2305, 2012
[3] Vipull, Virendra, Mayank, Ajith and Sugata Sanyal,"A New Protocol to Counter Online Dictionary Attacks", Computers and Security , Vol 25, no 2, Elsevier Science, pp. 114-120, 2006.

[4] Arfaat, "he Impact of Wormhole Attack on the Performance of Wireless Ad-Hoc Networks", pp. 421-425. , 2006.

[5] Badiwal, S, "Survey of IDS in MANET against Black Hole", vol.2, no.5, pp. 401-406, 2013.

[6] Bansal, P, "Impact of Black Hole and Neighbor Attack on AOMDV Routing Protocol", vol.3, no.4, pp. 90-99, 2012

[7] Jing e.t.al. :A study on fuzzy intrusion detection", Proceedings of SPIE: Data Mining, Intrusion Detection, Information Assurance, And Data Networks Security, vo.5812, pp. 23-30, 2005

[8] V. Jyothsna, Rama V. V. Prasad and Munivara K. Prasad, “A Review of Anomaly based Intrusion Detection Systems", International Journal of Computer Applications vol.28, no.7,, 2011, pp. 26-35.

[9] Jonathan Gomez, DipankarDasgupta, "Evolving Fuzzy Classifiers for Intrusion Detection", Proceeding of the 2002 IEEE, United States Military Academy, June 2001,

[10] Garcia Teodoro, Diaz Verdejo, Marcia Fernandez, Vazquez, “Anomaly-based network intrusion detection: Techniques, systems and challenges", Computer and Security, vol.28, pp. 18-28, 2009. https://doi.org/10.1016/j.cose.2008.08.003.

[11] Dhaval, Rajati, Punit Zalak and Dedhia, "Security scheme for distributed DoS in mobile ad hoc networks", May2010.

[12] Moreno and anchez. "GavabDB: a 3D facedatabase". In Workshop on Biometrics on the Internet, Vigo, 2004, pp.77-85.

[13] Bobor, "Efficient Intrusion Detection System Architecture Based on Neural Networks and Genetic Int. J. Advanced Networking and Applications", Volume: 03 Issue: 06 Pages: 1409-1415 (2012).

[14] Adel NadjaranToosi, Mohsen Kahani, "A newapproach to intrusion detection based on an evolutionary soft computing model using neurofuzzy classifiers", Computer Communications, vol.30, no.10, 2007, pp. 2201-2212 https://doi.org/10.1016/j.comcom.2007.05.002.

[15] Tabari, Hassanpour, and Movaghar, "Proposing a distributed model for intrusion detection in mobile ad-hoc network using neural fuzzy interface," in Journal of Advances in Computer Re- search, vol. 1, pp. 85-96, 2011.

[16] Wahengbam and Marchang, "Intrusion detec-tion in manet using fuzzy logic," in 3rd IEEE National Conference on Emerging Trends and Applications in Computer Science, pp. 189-192, 2012.

[17] Sen, Clark, and Tapiador, "Ad- hoc on-demand distance vector routing," in Security Threats in Mobile Ad Hoc Networks, Security of Self-Organizing Networks, pp. 127- 147, 2010.

[18] Shao, Lin, and Lee, "Cluster-based cooperative back propagation network approach for intrusion detection in MANET," in IEEE 10th In- ternational Conference on Computer an Information Technology, pp. 1627-1632, 2010.

[19] Moradi and Teshnehlab, "Intrusion detection model in manets using ANNs and ANFIS," in International Conference on Telecommunication Technol-ogy and Applications, vol. 5, 2011.

[20] moradi, Teshnehlab, and Rahmani, "Implementation of neural networks for intrusion detec-tion in MANET," in International Conference on Emerging Trends in Electrical and Computer Technology, pp. 1102-1106, 2011. 